\title{
Adenovirus-mediated siRNA targeting NOB1 inhibits tumor growth and enhances radiosensitivity of human papillary thyroid carcinoma in vitro and in vivo
}

\author{
WEI MENG, PEI-SONG WANG, JIA LIU, SHUAI XUE, GUI-MIN WANG, XIAN-YING MENG and GUANG CHEN \\ Department of Thyroid Surgery, The First Hospital of Jilin University, Changchun, Jilin 130021, P.R. China
}

Received June 26, 2014; Accepted August 12, 2014

DOI: $10.3892 /$ or.2014.3483

\begin{abstract}
NIN1/RPN12 binding protein 1 homolog (NOB1), a ribosome assembly factor, plays critical roles in tumor progression and development. Previously, we reported that overexpression of NOB1 is correlated with the prognosis of patients with papillary thyroid carcinoma (PTC). Little is known, however, concerning its role in PTC. The aims of the present study were to investigate the association of NOB1 expression with tumor growth and radiosensitivity of human PTC. A recombinant adenovirus expression vector carrying NOB1 was constructed and then infected into the human PTC cell line TPC-1. Cell proliferation, cell cycle distribution, apoptosis, migration and invasion in vitro and tumor growth in vivo were determined after downregulation of NOB1 by RNAi. Additionally, the in vitro and in vivo radiosensitivity of PTC cells was determined by clonogenic cell survival assay and a mouse xenograft model, respectively. The results showed that downregulation of NOB1 expression using RNAi in TPC-1 cells significantly inhibited cell proliferation, migration and invasion and induced cell apoptosis in vitro, and suppressed tumor growth in vivo, as well as enhanced the in vitro and in vivo radiosensitivity of PTC cells. Moreover, our results also showed that downregulation of NOB1 was able to significantly activate constitutive phosphorylation of p38 MAPK, which might contribute to the inhibition of PTC cell growth. These findings suggest that NOB1 may be a potential therapeutic target for the treatment of PTC.
\end{abstract}

\section{Introduction}

Thyroid cancer is the most common malignancy of the endocrine system and accounts for $\sim 1 \%$ of newly diagnosed cancers, and its incidence is rapidly increasing worldwide (1). Thyroid carcinoma can be divided into papillary, follicular,

Correspondence to: Professor Guang Chen, Department of Thyroid Surgery, The First Hospital of Jilin University, 71 Xinmin Street, Chaoyang District, Changchun, Jilin 130021, P.R. China

E-mail: chenguang518@aliyun.com

Key words: papillary thyroid carcinoma, NIN1/RPN12 binding protein 1 homolog, tumor growth, radiosensitivity medullary and anaplastic types according to histological type (2). Papillary is the most common thyroid carcinoma, accounting for more than $83 \%$ of all such malignancies $(3,4)$. Although the prognosis of patients with thyroid malignancies has improved as a result of the standardization of surgical techniques and advances in chemotherapy, a subset of patients suffers from recurrent disease that is refractory to surgical resection and radioactive iodine ablation $(5,6)$. Despite the fact that chemotherapy and radiotherapy have been widely used in the treatment of these patients, the outcome remains poor due to the intrinsic chemoresistance and radioresistance of papillary thyroid carcinoma (PTC) $(5,7)$. Therefore, there is a need to search for novel therapies to augment both systemic chemotherapy and radiotherapy for the treatment of patients with advanced PTC.

The NIN1/RPN12 binding protein 1 homologue (NOB1), an evolutionarily conserved protein, is a subunit of the $26 \mathrm{~S}$ proteasome and is composed of nine exons and eight introns 1,749 -bp long, containing a putative open reading frame of 1,239 bp, and is located on chromosome 16q22.1 (8). The NOB1 gene, encoding a 50-kDa protein consisting of a PilT $\mathrm{N}$ terminus (PIN) domain and a $\mathrm{C}$ terminal zinc ribbon domain $(9,10)$, is mainly expressed in liver, lung and spleen tissue (8). The PIN domain was postulated as the enzymatic domain of $N O B 1$ since cells expressing the mutant PIN failed to cleave the 20S pre-rRNA, strengthening the notion that NOB1 is the long-sought $\mathrm{D}$-site endonuclease $(11,12)$. As a ribosome assembly factor, recent biochemical and genetic studies have revealed that genetic depletion of Nob1 strongly suppresses the processing of the 20S pre-rRNA to the mature 18S rRNA, producing remarkably high levels of the $20 \mathrm{~S}$ pre-RNA with novel degradation intermediates (13). Additionally, a recent study also found that NOB1 plays a role in the formation of the $26 \mathrm{~S}$ proteasome and the maturation of the $20 \mathrm{~S}$ proteasome in eukaryotes (14). These above findings suggest that NOB1 plays a crucial role in the process of ribosome synthesis and the maturation and formation of the $26 \mathrm{~S}$ proteasome.

A larger amount of evidence suggests that the ribosome and the $26 \mathrm{~S}$ proteasomes are involved in the protein synthesis and ubiquitinated (ub) protein degradation pathways, respectively $(15,16)$. Numerous studies have demonstrated that the ubiquitin (Ub) pathway plays a critical role in regulating essential cellular processes, such as gene transcription and signal transduction $(17,18)$, which have close relationships with 
the development of human diseases, especially cancer (18-20). NOB1 is involved in the process of ribosome synthesis and the maturation and formation of the $26 \mathrm{~S}$ proteasome, which suggests a role for NOB1 in the development of human malignancies. $N O B 1$ has been found to be upregulated in a variety of cancers such as ovarian cancer, prostate carcinoma, breast infiltrating ductal carcinoma as well as in non-small lung cancer (21-24). Consistent with these findings, recently, our previous study demonstrated that the expression level of NOB1 protein was higher in PTC tissues than that in normal thyroid tissues and benign thyroid tumor tissues and there were significant associations between NOB1 protein expression and UICC stage, tumor size and lymph node metastasis (25). In particular, recently, several studies demonstrated that RNA interference (RNAi)-mediated downregulation of endogenous NOB1 inhibits tumor cell proliferation and survival, and induces cell apoptosis $(21,24,26,27)$. Radiation might kill cancer cells by inducing apoptosis, or programmed cell death, thus we hypothesized that reduction of NOB1 expression in PTC cells might enhance the radiosensitivity of cells by facilitating apoptotic pathways. However, the question of whether or not inhibition of NOB1 is an effective approach to overcome the radioresistance of PTC has not yet been explored. In addition, to our knowledge, whether NOB1 affects tumor cell proliferation or tumor growth of PTC remains unclear. Therefore, to solve these questions and explore the possibility of NOB1 as a therapeutic target for the treatment of human PTC, we first constructed an adenoviral vector plasmid to deliver small interfering RNA molecules targeting the NOB1 gene by the RNA interference (RNAi) technology. Then, cell proliferation, cell cycle distribution, apoptosis, migration and invasion in vitro and tumor growth in vivo were determined after downregulation of NOB1 by RNAi. Finally, the in vitro and in vivo radiosensitivity of PTC cells was evaluated.

\section{Materials and methods}

Cell culture. The human PTC cell lines (TPC-1) and HEK-293T, provided by the Chinese Cell Bank of the Chinese Academy of Sciences (Shanghai, China) were cultured in RPMI-1640 (Gibco, Carlsbad, CA, USA) containing 10\% fetal bovine serum (FBS; Gibco), $100 \mathrm{U} / \mathrm{ml}$ penicillin and $100 \mathrm{mg} / \mathrm{ml}$ streptomycin at $37^{\circ} \mathrm{C}$ in a humidified atmosphere of $5 \% \mathrm{CO}_{2}$.

siRNA design and production of the recombinant adenovirus. The siRNA target design tools from Ambion were used to design NOB1-shRNA and negative control scramble-shRNA sequences. shRNAs targeting NOB1 and the control sequence (Scramble) were designed and synthesized as follows: sh-NOB1 sense, 5'-GATCCAAGGTTAAGGTGAGCTCATCGTCA AGAGAAGTTGTACTCCAGCTTGTGAGA-3' and antisense, 5'-AGCTTAAGGTTAAGGTGAGCTCATCGCTCTTGAA AGTTGTACTCCAGCTTGTGG-3'; sh-Scramble sense, 5'-GATCCAATTCTCCGAACGTGTCACGTTCAAGAGAT CCGATTACGGCGTTTCTTAGA-3' and antisense, 5'-AGC TTAAGAAACGCCGTAATCGGATCTCTTGAATCCGAT TACGGCGTTTCTTG-3'. The oligonucleotides were annealed and inserted into the BamHI and HindIII sites of pSilencer 2.1-U6 neo (Ambion, Austin, TX, USA), and then subcloned into the HindIII and BglII sites of the pAd-Track adenoviral shuttle vector to get pAdTrack-U6/sh-NOB1 or pAdTrack-U6/ sh-Scramble, referred to as pAD-NOB1 and pAD-Scramble. Then, pAD-NOB1 or pAD-Scramble vectors were linearized with PmeI and cotransformed into BJ5183 cells with adenoviral backbone vector $\mathrm{pAdEasy-1.} \mathrm{Positive} \mathrm{clones} \mathrm{were} \mathrm{selected}$ and determined by DNA miniprep and PacI digestion. Plasmids from the correct clones were amplified by transforming into DH5K cells. Adenoviral DNA (pAD-NOB1 and pAD-Scramble) was prepared and linearized with PacI and purified by ethanol precipitation. The linearized recombinant plasmid was then transduced into the packaging cell line 293 as previously described $(28,29)$. pAD-NOB1 and pAD-Scramble adenovirus vectors were amplified using 293 cells, and titered using the Adeno- $\mathrm{X}^{\mathrm{TM}}$ Rapid Titer kit (BD Biosciences, San Jose, CA, USA) in 293 cells.

Adenovirus infection. On the day before virus infection, $3 \times 10^{5}$ TPC-1 cells were plated in each well of 6 -well plates. When the cells reached $\sim 70-90 \%$ confluency, the culture medium was aspirated, and the cell monolayer was washed with prewarmed sterile PBS ( $\mathrm{pH}$ 7.4). Cells were then incubated with the indicated virus (pAD-NOB1 and pAD-Scramble) at a multiplicity of infection (MOI) of $0,25,50$ or 75 at $37^{\circ} \mathrm{C}$, respectively. After adsorption for $2 \mathrm{~h}, 2 \mathrm{ml}$ of fresh growth medium was added, and the cells were placed in an incubator for an additional 48-72 h. Cell analysis and other experiments were performed. The following experiments were performed using viruses at such MOIs except for special indications.

Reverse transcription and real-time PCR. Total RNA from the cell samples was isolated by the TRIzol reagent (Invitrogen, Carlsbad, CA, USA) according to the manufacturer's instructions. Total RNA ( $2 \mathrm{mg}$ ) was reverse-transcribed into cDNA using M-MLV Reverse Transcriptase kit (Promega, Madison, WI, USA) according to the manufacturer's protocol. Real-time quantitative PCR analysis was performed using a SYBR-Green Master Mix Kit on a Bio-Rad Connect Real-Time PCR platform. In brief, each PCR reaction mixture, containing $10 \mu \mathrm{l}$ of 2X SYBR-GreenMaster Mix, $1 \mu$ l of sense and antisense primers $(5 \mu \mathrm{mol} / \mu \mathrm{l})$ and $1 \mu \mathrm{l}$ of cDNA (20 ng), was run for 40 cycles with denaturation at $95^{\circ} \mathrm{C}$ for $15 \mathrm{sec}$, annealing at $60^{\circ} \mathrm{C}$ for $15 \mathrm{sec}$ and extension at $72^{\circ} \mathrm{C}$ for $30 \mathrm{sec}$ in a total volume of $20 \mu \mathrm{l}$. For relative quantification, $2^{-\Delta \Delta \mathrm{Ct}}$ was calculated and used as an indication of the relative expression levels, which was calculated by subtracting the $\mathrm{Ct}$ values of the control gene from the $\mathrm{Ct}$ values of NOB1. The primer sequences for the PCR amplification of the NOB1 gene were 5'-AAGTGAGGAGGAGGAGGAG-3' and 5'-ACTTTCTTC AGGGTCTTGTTC-3'. The primers for housingkeeping gene GAPDH were forward, 5'-GAAGGTGAAGGTCGGAGTC-3' and reverse, 5'-GAAGATGGTGATGGGATTTC-3'.

Western blot analysis. TPC-1 cells were collected 5 days after infection with the adenovirus constructs, and total protein was isolated. Cells were harvested and washed with cold phosphate-buffered saline solution, and total proteins were extracted in the extraction buffer $[150 \mathrm{mM}$ sodium chloride, $50 \mathrm{mM}$ Tris hydrochloride ( $\mathrm{pH} 7.5$ ), $1 \%$ glycerol, $1 \%$ Nonidet-40 substitute solution] and quantified using the bicinchoninic acid (BCA) method. Equal amounts of protein 
(20 $\mu \mathrm{g}$ per lane) from the treated cells were loaded and electrophoresed on an 8-12\% sodium dodecyl sulfate (SDS) polyacrylamide gel and then electroblotted onto nitrocellulose membranes, blocked by $5 \%$ skim milk, and probed with the antibodies to NOB1 (Abcam, Cambridge, UK), and antibodies against phosphorylated (p-)p38MAPK (Cell Signaling Technology, Danvers, MA, USA), XIAP, Bcl-2, p38MAPK, p-ERK1/2, ERK1/2, JNK, p-JNK and GAPDH (Santa Cruz Biotechnology, Santa Cruz, CA, USA), respectively, followed by treatment with the secondary antibody conjugated with horseradish peroxidase (1:1,000; Santa Cruz Biotechnology). The proteins were detected by the enhanced chemiluminescence system and exposed to X-ray film. The absorbances of the positive bands in the analysis were measured by densitometry using a GIS Analysis system (Tannon, Shanghai, China).

Cell viability analysis. The MTT assay was used to determine the effect of the downregulation of NOB1 on the proliferation of cells. In brief, cells infected with $\mathrm{Ad} / \mathrm{sh}-\mathrm{NOB} 1$ or Ad/sh-Scramble (MOI of 50 each), along with untreated cells were seeded in a 96 -well plates at a density of $5 \times 10^{3}$ cells $/$ well. At indicated time points, $20 \mu 1$ methylthiazol tetrazolium (MTT) solution $(5 \mathrm{mg} / \mathrm{ml})$ was added into each well. After $4 \mathrm{~h}$ of incubation at $37^{\circ} \mathrm{C}, 150 \mu \mathrm{l}$ dimethyl sulfoxide (DMSO) was added to dissolve the crystals. After $10 \mathrm{~min}$ at room temperature, the absorbance was recorded at $570 \mathrm{~nm}$.

BrdU incorporation assay. Cells infected with Ad/sh-NOB1 or Ad/sh-Scramble (MOI of 50 each), along with the untreated cells, were cultured in 96 -well plates with $5 \times 10^{3}$ cells per well. A 5-bromodeoxyuridine (BrdU) incorporation assay was performed using the BrdU cell proliferation assay kit (Chemicon, Temecula, CA, USA) according to the manufacturer's instructions. The growth rate of cells was calculated as described previously (21).

Cell cycle analysis. Cells infected with Ad/sh-NOB1 or $\mathrm{Ad} / \mathrm{sh}$-Scramble (MOI of 50 each), along with the untreated cells, were cultured in 6-well plates. At the indicated time point, cells were harvested by centrifugation at 2,000 x $\mathrm{g}$ for 5 min, washed twice with PBS, and then fixed in ethanol. Then, cells were rehydrated and resuspended in PBS containing RNase A $(100 \mu \mathrm{g} / \mathrm{ml})$ on ice. After an additional incubation at room temperature for $30 \mathrm{~min}$, cells were stained with propidium iodide (PI) and were then analyzed by using a BD FACS Calibur Flow Cytometer (BD Biosciences, San Diego, CA, USA).

Apoptosis analysis. To measure the effect of adenovirus-mediated siRNA targeting NOB1 on cell apoptosis of TPC1 cells, TUNEL assay was performed. In briefly, cellular DNA fragmentation was measured with the ApoTag Red in situ apoptosis detection kit (Chemicon) according to the manufacturer's instructions when TPC1 cells were infected with Ad/sh-NOB1, or Ad/sh-Scramble for $48 \mathrm{~h}$. To quantify the apoptotic cells, the terminal deoxynucleotidyl transferase-mediated nick end labeling (TUNEL)-positive cells were counted using confocal microscopy (Olympus, Tokyo, Japan).

In addition, at the molecular level, we also detected other anti-apoptotic molecules, such as XIAP and Bcl-2 protein expression by western blotting as an additional indicator of apoptosis.

Cell migration assay. To assess the effect of the downregulation of NOB1 on cell migration, a wound-healing assay was performed. In brief, cells infected with Ad/sh-NOB1 or Ad/sh-Scramble at an MOI of 50, along with the untreated cells, were seeded into 24-well tissue culture plates. Fortyeight hours later, an artificial homogenous wound was scratched into the monolayer with a sterile plastic $100-\mu 1$ micropipette tip. After wounding, the debris was removed by washing the cells with serum-free medium. Migration of cells into the wound was observed at different time points. Cells that migrated into the wounded area or cells with extended protrusion from the border of the wound were visualized and photographed under an inverted phase-contrast microscope (Leica DMR, Germany).

Invasion analysis. The migration capacity of TPC1 cells was determined in vitro using Transwell Chambers (Corning, Tewksbury, MA, USA) in which the two chambers were separated by a Matrigel-coated polycarbonate membrane ( $8-\mu \mathrm{m}$ pore size). Cells infected with $\mathrm{Ad} / \mathrm{sh}-\mathrm{NOB} 1$ or Ad/sh-Scramble at an MOI of 50, along with the untreated cells, were seeded into cell culture inserts $(8-\mu \mathrm{m}$ pore size; Falcon; BD Bioscience) with $1 \times 10^{5}$ cells/well, precoated with $25 \mu \mathrm{l}$ of $20 \%$ Matrigel (2-3 $\mathrm{mg} / \mathrm{ml}$ protein), and then placed in a 24-well plate (Falcon). After cells had been cultured at $37^{\circ} \mathrm{C}$ for $40 \mathrm{~h}$, they were fixed and stained with $0.5 \%$ crystal violet. The cells on the top of the cell culture insert were removed by wiping with a cotton swab, and cell invasion was observed with an immunofluorescence microscope by counting the cells that had invaded into the bottom of the cell culture insert. All experiments were performed in triplicate.

Clonogenic cell survival assay. Cells infected with Ad/sh-NOB1 or Ad/sh-Scramble at an MOI of 50, along with the untreated cells, were plated into each well of 96-well plates with $5.0 \times 10^{3}$ cells/well. Seventy-two hours after plating, the cells were trypsinized, counted, and the appropriate number of cells were plated into 6-well plates and allowed to attach for $6 \mathrm{~h}$. Then, three types of TPC1 cells were irradiated with different doses of $6 \mathrm{MV}$ X-ray radiation by a 23-EX accelerator (Varian). The radiation doses were $0,2,4,6$, and $8 \mathrm{~Gy}$, respectively (the dose efficiency was $1.6 \mathrm{~Gy} / \mathrm{min}$ ) at room temperature and incubated for 14 days. Colonies were stained with crystal violet and colonies with more than 50 cells were counted. Plating efficiency was calculated as follows: Plating efficiency $=$ (clone number/total cell number $) \times 100 \%$. All experiments were repeated in triplicate. Finally, the cell survival fraction was determined and the cell survival curve was constructed.

Detection of caspase-3 activity. The caspase-3 activity was determined using the ApoAlert caspase-3 assay kit (Clontech, Mountain View, CA, USA) following the manufacturer's instructions. In brief, cells infected with $\mathrm{Ad} / \mathrm{sh}-\mathrm{NOB} 1$ or Ad/sh-Scramble at an MOI of 50, along with the untreated cells, were seeded at a density of $1.0 \times 10^{7}$ cells/well in a $6-\mathrm{cm}$ dish; $48 \mathrm{~h}$ later, after irradiation, the cells were lysed in $50 \mu \mathrm{l}$ 
lysis buffer provided in the assay kit and were incubated at $4^{\circ} \mathrm{C}$ for $30 \mathrm{~min}$. The enzyme activity in the supernatant was measured by cleavage of the substrate acetyl-Asp-GluVal-Asp-p-nitroanilidine (DEVD-pNA) to yield pNA. The relative caspase- 3 activity was measured as the absorbance at $405 \mathrm{~nm}$ via a microplate reader (Thermo Fisher Scientific Inc., Waltham, MA, USA). The results were calibrated relative to known concentrations of pNA and expressed as picomoles of substrate cleaved per min and per microgram of protein.

Tumor xenograft assay. All animal experiments were performed in accordance with institutional guidelines, following a protocol approved by the Ethics Committees of the Disease Model Research Center, The First Hospital of Jilin University (Changchun, China). Female BALB mice $\sim 6$ weeks old were provided by the Disease Model Research Center, The First Hospital of Jilin University, and maintained under specific pathogen-free conditions and provided with food and water ad libitum.

The female BALB/c nude mice were inoculated subcutaneously with a total of $1.0 \times 10^{7} \mathrm{TPC} 1$ cells in $100 \mu \mathrm{l}$ of PBS into the right flank. Three weeks after tumor inoculation with the TCP1 cells, when the tumors grew to $\sim 100 \mathrm{~mm}^{3}, 60$ tumor-bearing mice were randomly divided into the following 4 treatment groups ( $\mathrm{n}=10 /$ group): i) PBS group (Control group); ii) Ad/sh-Scramble; iii) Ad/sh-NOB1; iv) irradiation; v) Ad/sh-Scramble + irradiation; vi) Ad/sh-NOB1 + irradiation. Three of the groups were irradiated, and three groups remained non-irradiated. Concerning the irradiated groups, intratumoral injections of $0.1 \mathrm{ml}$ of PBS, $6.0 \times 10^{8} \mathrm{pfu}$ of pAd-sh-Scramble or pAd-sh-NOB1 were administered three times on days 1,3 and 5 , and subsequently $\mathrm{X}$-ray irradiation was performed at a clinically relevant dose of $5.0 \mathrm{~Gy}$ on days 2, 4 and 6 . For the non-irradiated groups, intratumoral injections of $0.1 \mathrm{ml}$ of PBS, $6.0 \times 10^{8} \mathrm{pfu}$ of Ad/sh-Scramble or Ad/sh-NOB1 were conducted. Tumors were measured using a caliper gauge once a week over a 5-week period following the initial virus injections. Tumor volume was calculated according to the formula: TV $\left(\mathrm{mm}^{3}\right)=$ length $\mathrm{x}$ width ${ }^{2} \mathrm{x} 0.4$. All mice were sacrificed and tumors were resected and weighed, and part of the tumors was fixed in $10 \%$ PBS for the TUNEL assay. We measured the primary tumors and performed western blot analyses for NOB1 protein expression. Then, TUNEL staining was performed on $5-\mu \mathrm{m}$ sections of the excised tumors using Dead End ${ }^{\mathrm{TM}}$ Fluorometric TUNEL system (Promega) according to the manufacturer's protocol. The number of apoptotic cells in 5 random high-power fields was counted and expressed as a percentage of total cells (apoptotic fraction).

Statistical analysis. All data are expressed as mean \pm standard deviation (SD) from three independent experiments. Statistical analysis between two samples was performed using the Student's t-test. Statistical comparison of more than two groups was performed using one-way ANOVA followed by a Tukey post hoc test. Graphpad Prism 6.0 software (GraphPad Software, San Diego, CA, USA), was used for statistical analyses. $\mathrm{P}<0.05$ was considered to indicate a statistically significant difference.

\section{Results}

Adenovirus-mediated siRNA targeting NOBI inhibits the expression of NOB1 in TPCl cells. To determine the optimal MOI for maximal transgene expression, TPC1 cells were infected with $\mathrm{Ad} / \mathrm{sh}$-Scramble or Ad/sh-NOB1 at different MOIs $(0,25,50,75)$ and examined by fluorescence microscopy. Approximately $90 \%$ of GFP expression could be observed in the TPC1 cells infected with Ad/sh-Scramble or Ad/sh-NOB1 at an MOI of 50 (data not shown). Thus, an MOI of 50 was selected as an optimal dose for infection of the TPC1 cells and were used subsequently in all procedures. To determine the effect of the adenovirus-mediated siRNA targeting NOB1 on the expression of the NOB1 gene in the PTC cell line, real-time RT-PCR and western blot assay were performed to detect the expression of NOB1 at the mRNA and protein levels, respectively. The expression level of NOB1 mRNA in the Ad/sh-NOB-infected TPC1 cells was decreased compared to that in the untreated cells or Ad/sh-Scramble-infected cells $(\mathrm{P}<0.05$; Fig. $1 \mathrm{~A})$. Additionally, at the protein expression level, no significant inhibition in NOB1 protein expression was found in the untreated cell or Ad/sh-Scramble-infected cells $(\mathrm{P}>0.05)$, while the band density decreased dramatically in the Ad/sh-NOB1-infected TPC1 cells as compared with the uninfected cell or the Ad/sh-Scramble-infected cells $(\mathrm{P}<0.05$; Fig. 1B). These data revealed that adenovirus-mediated siRNA targeting NOB1 specifically and significantly inhibited the expression of the $N O B 1$ gene in the TPC 1 cells.

Adenovirus-mediated siRNA targeting NOB1 inhibits cell growth in TPC1 cells. To further assess the role of NOB1 in regulating PTC cell proliferation, MTT assays were performed on TPC1 cells following adenoviral infection for 5 days. Fig. 2A shows that there was no statistically significant difference in viability between the non-infected cells and the cells infected with Ad/sh-Scramble, indicating that the adenoviral system itself had no cytotoxic effect on cells. In contrast, the viability of the TPC 1 cells was markedly inhibited following NOB1 knockdown $(\mathrm{P}<0.05$ compared to control), and the inhibitory effect of Ad/sh-NOB1 on cell proliferation was observed beginning on day 2; it became more obvious on days 4 and 5 ( $\mathrm{P}<0.05$; Fig. 2A). Moreover, BrdU incorporation assays also revealed that the inhibition of NOB1 expression significantly reduced the growth rate of TPC1 cells during the $48-\mathrm{h}$ incubation period $(\mathrm{P}<0.05$; Fig. 2B). These findings suggest that the knockdown of NOB1 greatly diminished the cell proliferative ability in the TPC1 cells.

Adenovirus-mediated siRNA targeting NOB1 induces cell $G_{0} / G_{I}$ phase and apoptosis. Knowing that the inhibition of NOB1 in TPC1 cells markedly slows cell proliferation, we further employed cell-cycle analysis to reveal the mechanism governing the inhibitory effect of the downregulation of NOB1 on cell proliferation. As shown in Fig. 3A, in TPC1 cells, an obvious increase in the $\mathrm{G}_{1}$-phase cell population was observed in the $\mathrm{Ad} / \mathrm{sh}-\mathrm{NOB} 1$ group as compared with this population in the $\mathrm{Ad} / \mathrm{sh}$-Scramble and control groups $(\mathrm{P}<0.05)$. Our results suggest that Ad/sh-NOB1 exerted an inhibitory effect on TPC1 cell proliferation via $\mathrm{G}_{0} / \mathrm{G}_{1}$. 
A

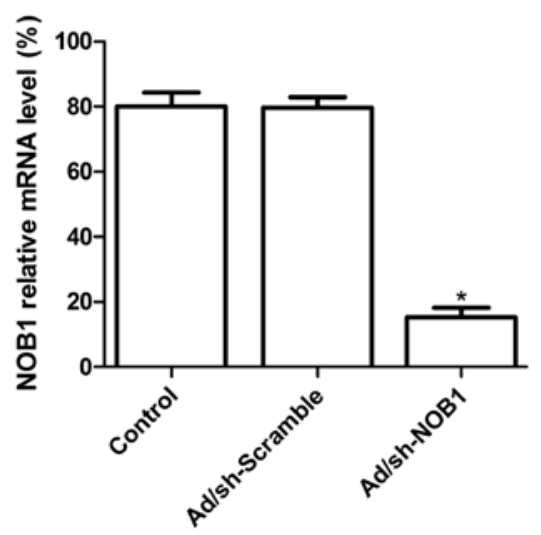

B

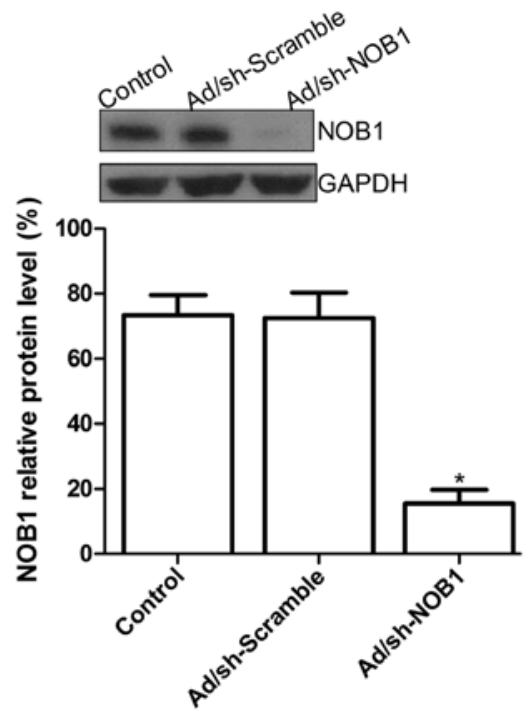

Figure 1. Determination of NOB1 mRNA and protein expression by real-time RT-PCR and western blot assay, respectively. (A) Real-time RT-PCR analysis of NOB1 mRNA expression in TPC1 cells after infection with Ad/sh-NOB1 or Ad/sh-Scramble. Data were collected from three independent experiments. (B) Western blot analysis of NOB1 protein levels in TPC1 cells after infection with Ad/sh-NOB1 or Ad/sh-Scramble (GAPDH was included as an internal control). Data were collected from three independent experiments; ${ }^{*} \mathrm{P}<0.05$ vs. the control group.

A

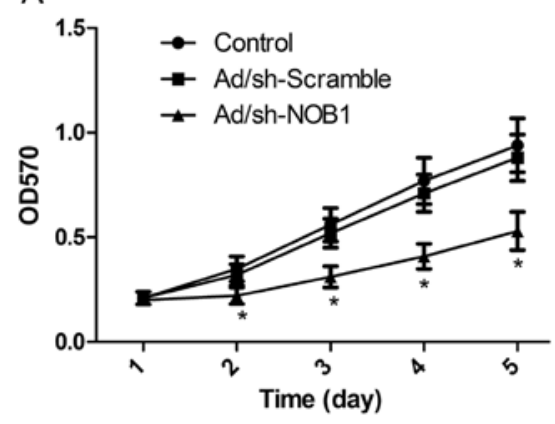

B

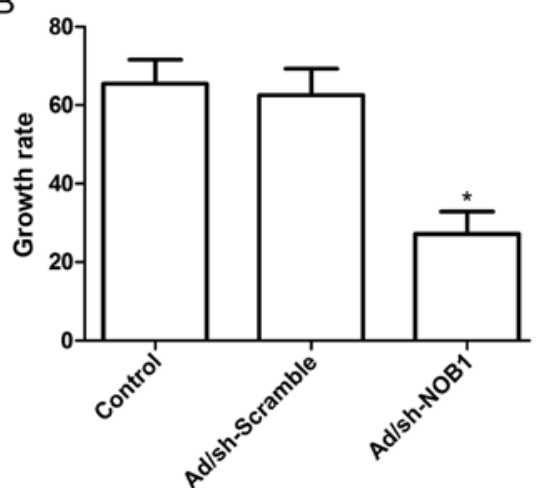

Figure 2. Downregulation of NOB1 by an adenovirus-mediated siRNA inhibits TPC1 cell proliferation. (A) NOB1 silencing by shRNA adenovirus resulted in growth inhibition as detected by MTT assay in TPC1 cells. Cells infected with Ad/sh-NOB1 or Ad/sh-Scramble for 5 days, along with the untreated cells, were seeded in a 96-well plates, and cell viability was determined at the indicated times. (B) NOB1 silencing by shRNA adenovirus led to TPC1 cell growth inhibition as detected by a BrdU incorporation assay. The growth rate of TPC1 was calculated. All assays were performed in triplicate. "P<0.05 vs. the control.

Next, to further investigate the effect of the adenovirusmediated siRNA targeting NOB1 on cell apoptosis in TPC1 cells, cell apoptosis was analyzed by TUNEL. Compared with the control group and Ad/sh-Scramble group, the Ad/sh-NOB1 group underwent significantly induced cell apoptosis $(\mathrm{P}<0.05$; Fig. 3B). Next, we analyzed the effects of adenovirus-mediated siRNA targeting NOB1 on the expression of other apoptosis relevant proteins including Bcl-2 and XIAP. As shown in Fig. $3 \mathrm{C}$ and D, the levels of $\mathrm{Bcl}-2$ and XIAP protein expression in the cells infected with Ad/sh-NOB1 showed a significant decrease compared with the levels in the control group and $\mathrm{Ad} /$ sh-Scramble cells $(\mathrm{P}<0.05$; Fig. 3D). These results suggest that the adenovirus-mediated siRNA targeting NOB1 can induce cell $\mathrm{G}_{0} / \mathrm{G}_{1}$ arrest and apoptosis in TPC1 cells.

Adenovirus-mediated siRNA targeting NOB1 inhibits cell migration and invasion in TPC1 cells. To ascertain the inhibi- tory effect of the adenovirus-mediated siRNA targeting NOB1 on thyroid cancer cell motility in vitro, a wound-healing assay was performed to investigate the effects on the migration potential of PTC cells. A scratch was introduced into confluent monolayers of the cells expressing the different treatment plasmids, and the time-dependent movement of cells into the injured area was monitored microscopically. Cells in the control and Ad/sh-Scramble groups began migrating $8 \mathrm{~h}$ after scratching. After $48 \mathrm{~h}$, cells in the Ad/sh-NOB1 group migrated significantly less rapidly than those in the control and the Ad/sh-Scramble groups ( $\mathrm{P}<0.05$; Fig. 4A and B).

Next, the ability of adenovirus-mediated siRNA targeting NOB1 to reduce the invasiveness of the PTC cells was further investigated using the Transwell system assay. Invasion was also decreased significantly in the Ad/sh-NOB1 treatment group compared to the control and the Ad/sh-Scramble groups $(\mathrm{P}<0.05$; Fig. 4C and D). 

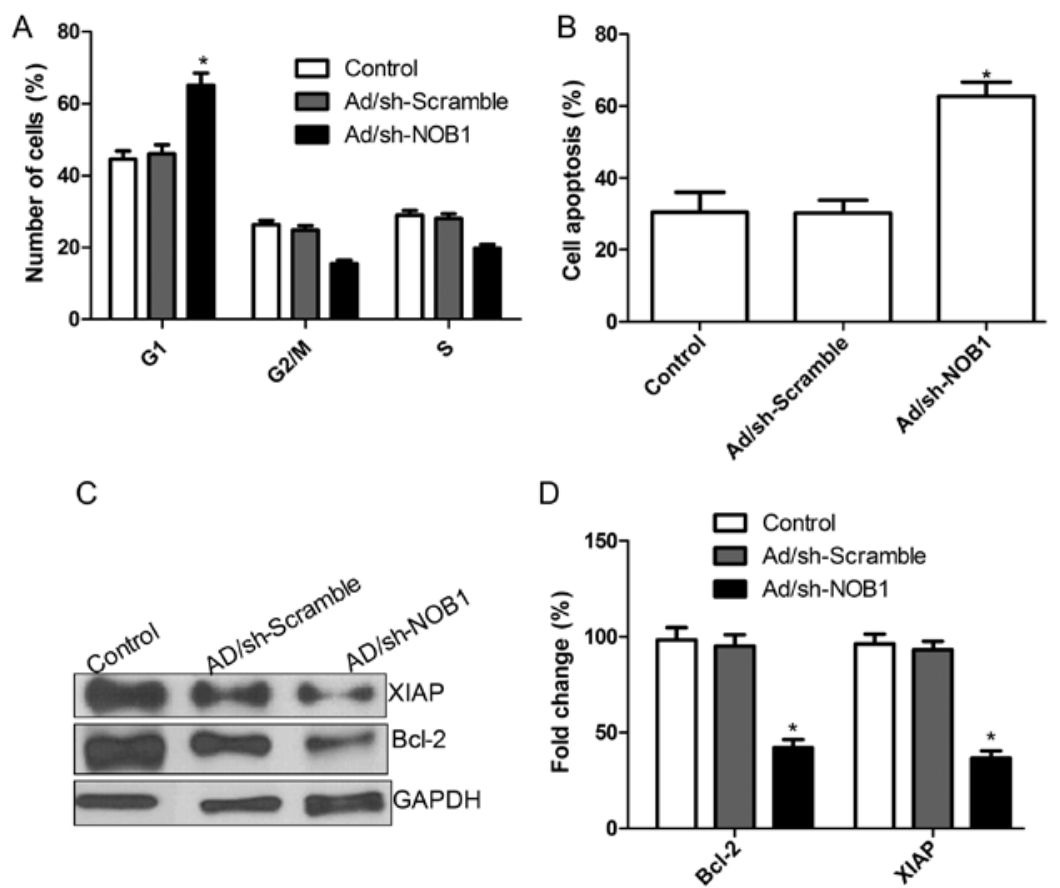

Figure 3. Downregulation of NOB1 by adenovirus-mediated siRNA induces $G_{0} / G_{1}$ phase arrest and cell apoptosis in TPC1 cells. (A) Quantification of the percentage of cells in cell cycle phases $\mathrm{G}_{1}, \mathrm{~S}$ and $\mathrm{G}_{2} / \mathrm{M}$. In the Ad/sh-NOB1 group, a significant increase in the $\mathrm{G}_{1}$ phase cell population and an obvious decrease in the $\mathrm{G}_{2} / \mathrm{M}$ phase cell popuation was observed, as compared with the control group and Ad/sh-Scramble group ( $\mathrm{P}<0.05$ ). (B) TUNEL analysis of TPC1 cell apoptosis after infection with Ad/sh-NOB1 or Ad/sh-Scramble. Cell apoptosis of TPC1 cells significantly increased in the Ad/sh-NOB1 group compared to the control group (" $\mathrm{P}<0.05)$. (C) Western blot analysis of Bcl-2 and XIAP protein expression after infection with Ad/sh-Scramble or Ad/sh-NOB1. GAPDH was used as an internal control. Each assay was performed at least in triplicate. (D) Relative quantification of Bcl-2 and XIAP protein by densitometric analysis. ${ }^{*} \mathrm{P}<0.05$ vs. the control.

A

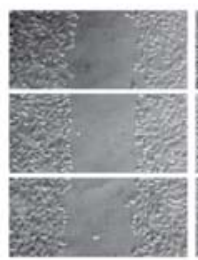

$\mathrm{Oh}$

C

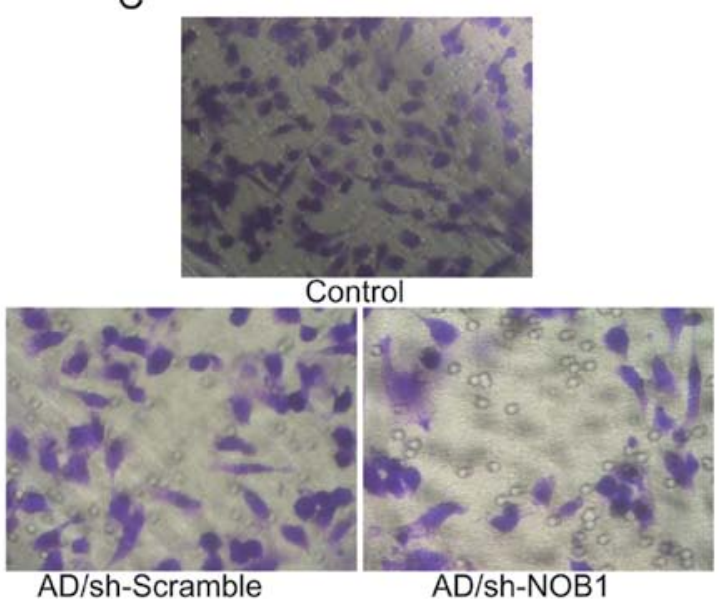

$\mathrm{B}$

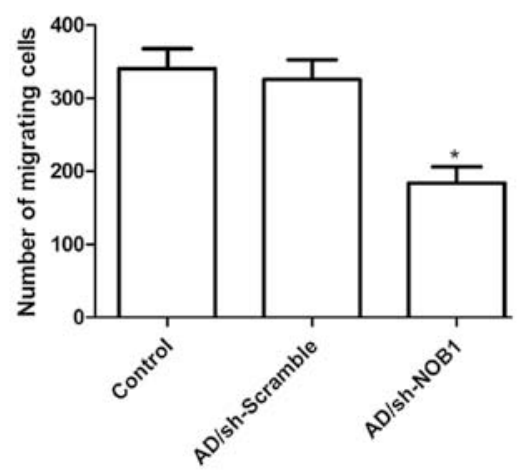

D

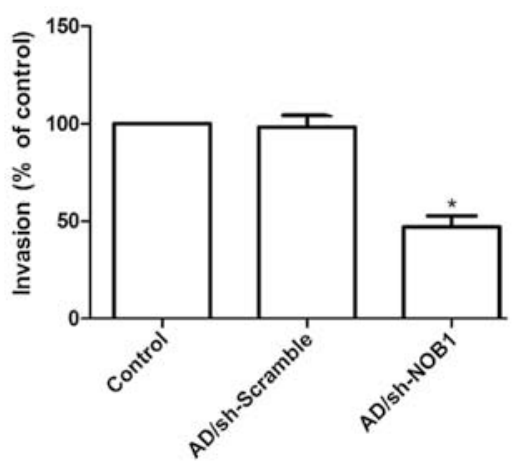

Figure 4. Downregulation NOB1 by adenovirus-mediated siRNA inhibits cell migration and invasion. (A) Cell migration was determined by a wound-healing assay after infection of TPC1 cells with Ad/sh-Scramble or Ad/sh-NOB1. Phase micrographs of cells were taken at 0 and $48 \mathrm{~h}$ after monolayer wounding. (B) The number of migrating cells was counted $48 \mathrm{~h}$ after infection with Ad/sh-Scramble or Ad/sh-NOB1. (C) Cell invasion was determined by Matrigel in TPC1 cells after infection with Ad/sh-Scramble or Ad/sh-NOB1. (D) Histogram of invaded cells in each group, normalized to the control cells, after infection with Ad/sh-Scramble or Ad/sh-NOB1. "P<0.05 vs. control. 
Table I. Plating efficiencies at different radiation doses.

\begin{tabular}{lccccc}
\hline & \multicolumn{5}{c}{ Plating efficiency } \\
\cline { 2 - 6 } Cell & 0 Gy & 2 Gy & 4 Gy & 6 Gy & 8 Gy \\
\hline TPC1 cell & $95.49 \pm 4.16$ & $83.45 \pm 3.06$ & $65.88 \pm 2.41$ & $46.42 \pm 1.58$ & $24.42 \pm 1.06$ \\
Ad/sh-Scramble & $96.54 \pm 3.68$ & $81.46 \pm 3.01$ & $63.90 \pm 1.78$ & $47.28 \pm 1.29$ & $20.84 \pm 0.76$ \\
Ad/sh-NOB1 & $89.58 \pm 3.38$ & $56.66 \pm 1.84^{\mathrm{a}}$ & $34.21 \pm 1.76^{\mathrm{a}}$ & $12.89 \pm 0.58^{\mathrm{a}}$ & $5.34 \pm 0.41^{\mathrm{a}}$ \\
\hline
\end{tabular}

Values are expressed as mean $\pm \mathrm{SD}(\%) .{ }^{\mathrm{a}} \mathrm{P}<0.05$ vs. the TPC 1 cells.

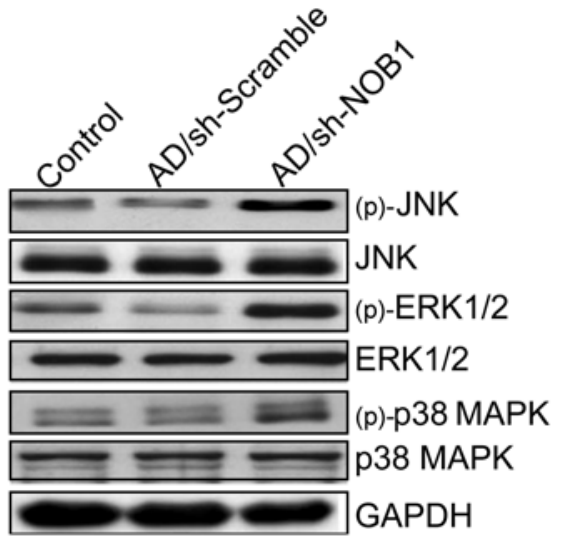

Figure 5. Effect of the downregulation of NOB1 by adenovirus mediated siRNA on the MAPK signaling pathway in TPC1 cells. Phosphorylation/ activation pattern of $\mathrm{p} 38$ MAPK, ERK1/2 and JNK was measured by western blotting $24 \mathrm{~h}$ after infection with Ad/sh-Scramble or Ad/sh-NOB1 in the TPC1 cell line. Blots were reprobed for GAPDH to normalize each lane for protein content.

Adenovirus-mediated siRNA targeting NOBI activates the MAPK signaling pathway. To clarify the molecular mechanisms involved in the inhibition of cell proliferation and survival of PTC cells following downregulation of NOB1, in the present study, we investigated the effects of adenovirusmediated siRNA targeting NOB1 on the activation of the MAPK pathway, which participates in the main intracellular signaling required for cell proliferation and survival. Measurements of the phosphorylation/activation pattern of p38 MAPK, ERK1/2 and JNK were performed by western blotting. Our results showed that adenovirus-mediated siRNA targeting NOB1 resulted in a marked increase in phosphorylated (p)-p38 MAPK, (p)-ERK1/2 and (p)-JNK relative to the levels in the control group and $\mathrm{Ad} / \mathrm{sh}$-Scramble group, without altering the total protein levels of p38 MAPK, ERK1/2 and JNK in each group (Fig. 5). These results indicate that adenovirus-mediated siRNA targeting NOB1 inhibits TPC1 cell proliferation and induces apoptosis, to some extent, by activating the MAPK signaling pathway.

Adenovirus-mediated siRNA targeting NOBI enhances the radiosensitivity of TPCl cells. To explore the effect of adenovirus-mediated siRNA targeting NOB1 on the radiosensitivity of TPC1 cells, a clonogenic cell survival assay was performed. As shown in Table I, the plating efficiencies of $\mathrm{Ad} /$ sh-NOB1 cells at the same doses of radiation were significantly decreased compared with those of the control cells and the Ad/sh-Scramble group. Compared with the uninfected TPC1 cells, the cell survival curve obviously decreased in the Ad/ sh-NOB1 group $(\mathrm{P}<0.05$; Fig. $6 \mathrm{~A})$, whereas the cell survival curve in the $\mathrm{Ad} / \mathrm{sh}-\mathrm{Scramble}$ group decreased but without significance (Fig. 6A).

To determine the activation of the caspase cascade during the process of apoptosis, we detected the changes in caspase- 3 activity in the TPC1 cells treated with radiation alone, radiation combination with $\mathrm{Ad} / \mathrm{sh}$-Scramble or radiation combined with Ad/sh-NOB1. Results from the colorimetric assay showed that the caspase-3 activity in TPC1 cells treated with radiation combined with the Ad/sh-NOB1 was increased compared with the $\mathrm{TPCl}$ cells treated with radiation alone or radiation combined with Ad/sh-Scramble (Fig. 6B; P<0.05). These results imply that the downregulation of NOB1 expression leads to radiosensitivity of thyroid cancer cells by activating celluar caspase-3, but the exact mechanism needs to be further investigated.

Adenovirus-mediated siRNA targeting NOB1 inhibits tumor growth and enhances the radiosensitivity of TPCl cells in vivo. Finally, we assessed the in vivo therapeutic efficacy of adenovirus-mediated siRNA targeting NOB1 on female BALB mice bearing TPC1 cell tumors. Tumor growth was monitored for 36 days. On day 36, the animals were sacrificed, and then tumors were excised, weighed and measured. Tumor weights were significantly lower in the various treatment groups than those in the control group (PBS group) and $\mathrm{Ad} / \mathrm{sh}$-Scramble group $(\mathrm{P}<0.05$; Fig. 7A). Compared to the other groups, the Ad/sh-NOB1 in combination with irradiation group showed a maximally reduced weight. In addition, tumor volume was also determined at different times. The tumor volumes in the various treatment groups were significantly $(\mathrm{P}<0.05)$ diminished when compared with the Ad/ sh-Scramble group and control group (PBS group) at different times $(\mathrm{P}<0.05$; Fig. 7B). Importantly, Ad/sh-NOB1 combined with irradiation led to a significant inhibition of tumor volume compared with the other treatment groups $(\mathrm{P}<0.05$; Fig. 7B). At the same times, we also examined the expression of NOB1 in grafted tumor tissues by western blot analysis. As shown in Fig. 7C, the expression of NOB1 protein in the tumors in the $\mathrm{Ad} / \mathrm{sh}-\mathrm{NOB} 1$-treated group was significantly decreased compared with the PBS group or Ad/sh-Scramble group. Finally, we also determined the synergistic effects on tumor tissue cell apoptosis in vivo by TUNEL. The results showed that Ad/sh-NOB1 and irradiation alone or the combination 
A

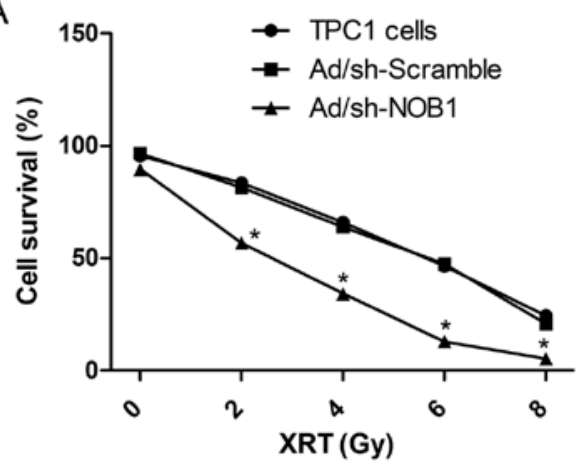

B

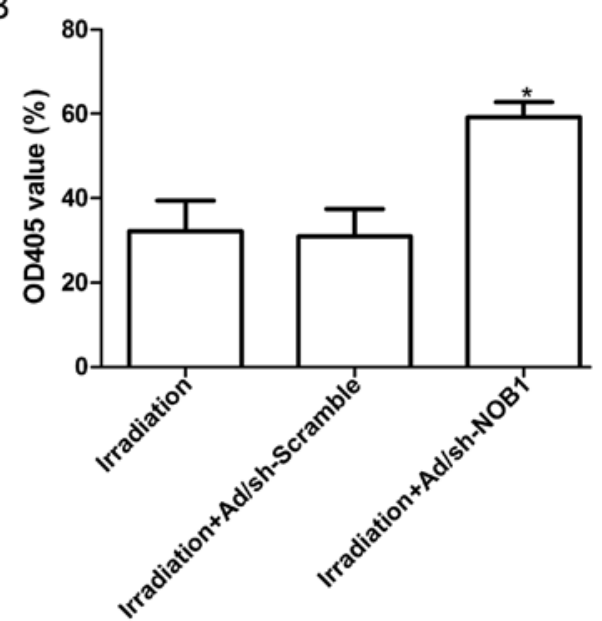

Figure 6. Effects of the downregulation of NOB1 by adenovirus-mediated siRNA on the in vitro sensitivity of TPC1 cells to irradiation. (A) Clonogenic survival after varying doses of irradiation exposure. The control, Ad/sh-Scramble- or Ad/sh-NOB1-infected TPC1 cells were irradiated followed by further incubation for $24 \mathrm{~h}$ at $37^{\circ} \mathrm{C}$ before trypsinization and plating for clonogenic survival. After 10-14 days of incubation, colonies were stained, and the surviving fraction was determined. (B) Caspase-3 activities in TPC1 cells. After the cells were treated with Ad/sh-NOB1 or Ad/sh-Scramble along with irradiation or irradiation alone, caspase- 3 activities were determined. ${ }^{*} \mathrm{P}<0.05$ vs. the cell group treated with irradiation alone.

A

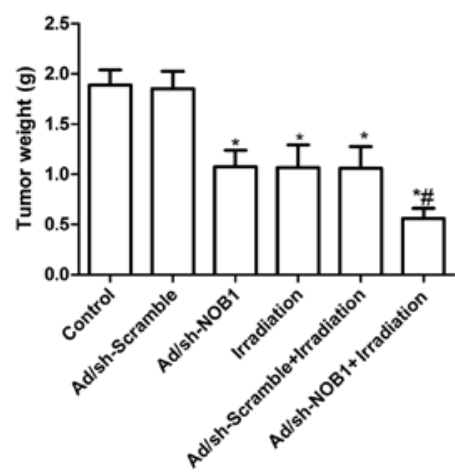

C

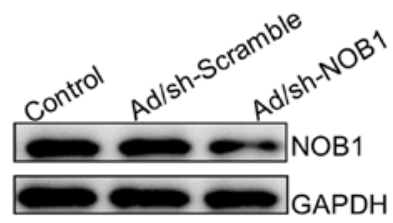

B

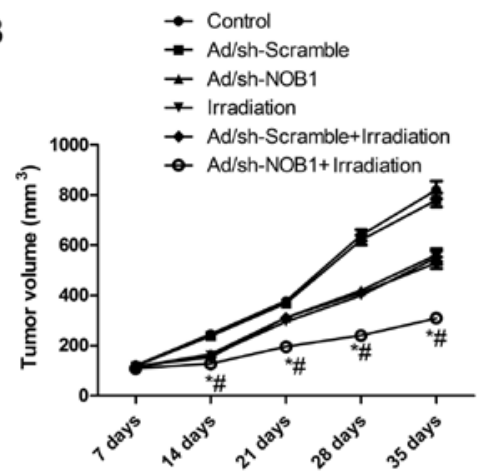

$\mathrm{D}$

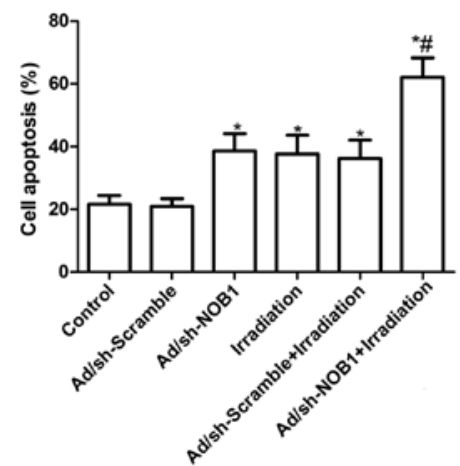

Figure 7. Effect of the downregulation of NOB1 by adenovirus-mediated siRNA on the radiosensitivity of papillary thyroid carcinoma cells in vivo. (A) Average tumor weight at day 36 after treatment with Ad/sh-NOB1 and irradiation alone or both. (B) Average tumor volume at different times after treatment with Ad/sh-NOB1 and irradiation alone or both. (C) Protein samples extracted from TPC1 tumors (n=10/group) were analyzed using western blot analysis for NOB1 expression levels. GAPDH was included as a loading control. (D) TUNEL assay of cell apoptosis in vivo. Data are expressed as the mean values \pm SD. ${ }^{*} \mathrm{P}<0.05$ vs. the control; ${ }^{\mathrm{P}}<0.05$ vs. the cell group treated with irradiation alone.

treatment significantly induced cell apoptosis compared to that noted in the control group and the Ad/sh-Scramble groups $(\mathrm{P}<0.05$; Fig. 7D). The combination of Ad/sh-NOB1 and irradiation greatly induced tumor cell apoptosis in vivo compared to the single treatment groups $(\mathrm{P}<0.05$; Fig. 7D).
These experimental data suggest that adenovirus-mediated siRNA targeting NOB1 could increase the in vivo radiosensitivity of PTC cells, which might imply that Ad/sh-NOB1 combined with radiotherapy leads to a stronger antitumor effect on human PTC. 


\section{Discussion}

The majority of patients with papillary thyroid cancer (PTC) have a favorable prognosis, yet a subgroup of patients suffer from recurrent disease that is refractory to surgical resection and radioactive iodine ablation (6). Unfortunately, no effective systemic treatment exists for these patients. The conventional chemotherapy and radiotherapy regimens are more of a hindrance due to their limited efficacy, significant toxicity, the development of resistance and high relapse rates (30). Newer therapeutic approaches to solve this issue are required. Therefore, further studies investigating multiple treatment modalities and/or the inhibition of multiple signaling pathways are required in our efforts to develop a novel therapeutic strategy to enhance both systemic chemotherapy and radiotherapy.

NOB1, identified as an interacting partner with RPN12p by a yeast two-hybrid screening, is a nuclear protein that forms a complex with the $19 \mathrm{~S}$ regulatory particle of the $26 \mathrm{~S}$ proteasome, and takes part in processing the $20 \mathrm{~S}$ prerRNA to the mature 18S rRNA (31). It has been shown that the $26 \mathrm{~S}$ proteasome catalyzes protein degradation via the ubiquitin-proteasome system (UPS), which is required for the degradation of cyclic proteins and regulates multiple aspects of the cell cycle progression in eukaryotes $(32,33)$. UPS plays a critical role in cancer development and progression. In light of the important role of NOB1 and the critical function of ubiquitin-dependent proteolysis in universal biological processes, recently, several studies have focused on the role of NOB1 in tumor development and progression (21-27). For example, Li et al showed that downregulation of NOB1 expression using an RNA silencing approach in A549 tumor cells significantly suppressed the proliferation and colony formation ability, and induced tumor apoptosis in vitro, and suppressed tumor growth in vivo (24). Lin et al demonstrated that RNA interference (RNAi)-mediated downregulation of NOB1 expression markedly reduced the proliferative and colony-formation ability of ovarian cancer cells (21). Wang et al found that the expression level of the NOB1 protein was significantly higher in high-grade gliomas than in low-grade gliomas, and that knockdown of the NOB1 gene resulted in suppression of the proliferation and the colony forming abilities of U251 and U87-MG cells, cell cycle arrest during the $\mathrm{G}_{0} / \mathrm{G}_{1}$ phase, and a significant enhancement of cell apoptosis (34). Consistent with these results, our results showed that downregulation of NOB1 expression using adenovirus-mediated siRNA targeting NOB1 in TPC-1 cells significantly inhibited cell proliferation, migration and invasion and induced cell apoptosis in vitro, as well as suppressed tumor growth in a mouse model. These studies and our results suggest that NOB1 plays critical roles in tumor progression and development.

The MAPKs are serine/threonine protein kinases that are involved in intracellular signaling during proliferation, differentiation, cellular stress responses and apoptosis (35). MAPK signaling is mediated by extracellular signal regulated kinase 1 and 2 (ERK1/2), p38 MAPK, and the stress activated protein kinase (SAPK)/c-Jun NH2-terminal kinase (JNK), which are important in the control of cell proliferation, differentiation and apoptosis (36). The activation of the MAPK pathway has been implicated in the activity of numerous chemotherapy and genotoxic drugs (37). In addition, activation of the MAPK pathway plays an important role in radiotherapy by mediation or regulation of cellular responses such as proliferation, migration and even transformation/carcinogenesis (38). Importantly, it was recently shown that silencing of NOB1 expression increased the phosphorylation of ERK1/2, JNK and p38 MAPK proteins, and that the anti-glioma effect of NOB1 might be mediated by MAPK activation (39). However, Chen et al found that NOB1 expression in prostate cancer was independently associated with p38 MAPK activation, and that p38 MAPK expression was completely suppressed by NOB1 interference in the prostate cancer cell lines DU-145 and PC-3 (22). These controversial findings were found since the role of MAPKs in certain functions is controversial and complicated, and depends on the stimuli, intensity, and duration, as well as cell type (40). In the present study, our results showed that treatment with an adenovirus-mediated siRNA targeting NOB1 resulted in a marked increase in phosphorylated (p)-p38 MAPK, ERK1/2 and JNK relative to the control group and Ad/sh-Scramble group, without altering the total protein levels of p38 MAPK, ERK1/2 and JNK in each group, suggesting that activation of the MAPK pathway may play an important role in PTC cell proliferation, apoptosis and migration. In addition, our experimental data showed that adenovirus-mediated siRNA targeting NOB1 could increase the in vitro and in vivo radiosensitivity of PTC cells. In light of the important role of the MAPK signaling pathway and the critical function of radiotherapy, we assume that downregulation of NOB1 may influence the radiosensitivity of PTC cells through activation of the MAPK pathway.

In conclusion, we demonstrated for the first time that an adenovirus-mediated siRNA targeting NOB1 in human PTC cells inhibits cell proliferation, migration and invasion in vitro, and suppresses tumor growth in a mouse model, as well as it enhances in vitro and in vivo radiosensitivity of PTC cells. Moreover, our results also showed that downregulation of NOB1 was able to significantly activate the phosphorylation of p38 MAPK, which might contribute to the inhibition of papillary PTC cell growth. These results suggest that NOB1 appears to have therapeutic potential for the treatment of PTC.

\section{Acknowledgements}

This research was supported by the Science and Technology Research and Innovation Team funded by Jilin Province (JL20130518).

\section{References}

1. Brown RL, de Souza JA and Cohen EE: Thyroid cancer: burden of illness and management of disease. J Cancer 2: 193-199, 2011.

2. Thompson L: World Health Organization classification of tumours: pathology and genetics of head and neck tumours. Ear Nose Throat J 85: 74, 2006.

3. Nikiforova MN and Nikiforov YE: Molecular genetics of thyroid cancer: implications for diagnosis, treatment and prognosis. Expert Rev Mol Diagn 8: 83-95, 2008.

4. Dal Maso L, Bosetti C, La Vecchia C and Franceschi S: Risk factors for thyroid cancer: an epidemiological review focused on nutritional factors. Cancer Causes Control 20: 75-86, 2009.

5. Jeong SY, Kim HW, Lee SW, Ahn BC and Lee J: Salivary gland function 5 years after radioactive iodine ablation in patients with differentiated thyroid cancer: direct comparison of pre- and postablation scintigraphies and their relation to xerostomia symptoms. Thyroid 23: 609-616, 2013. 
6. Matuszczyk A, Petersenn S, Bockisch A, et al: Chemotherapy with doxorubicin in progressive medullary and thyroid carcinoma of the follicular epithelium. Horm Metab Res 40: 210-213, 2008.

7. Strasser JF, Raben A and Koprowski C: The role of radiation therapy in the management of thyroid cancer. Surg Oncol Clin N Am 17: 219-232, 2008.

8. Zhang Y, Ni J, Zhou G, et al: Cloning, expression and characterization of the human NOB1 gene. Mol Biol Rep 32: 185-189, 2005.

9. Makarova KS, Aravind L and Galperin MY: Comparative genomics of the Archaea (Euryarchaeota): evolution of conserved protein families, the stable core, and the variable shell. Genome Res 9: 608-628, 1999.

10. Arcus VL, Backbro K, Roos A, Daniel EL and Baker EN: Distant structural homology leads to the functional characterization of an archaeal PIN domain as an exonuclease. J Biol Chem 279: 16471-16478, 2008.

11. Lamanna AC and Karbstein K: Nob1 binds the signal-stranded cleavege site D at the 3'-end of 18S rRNA with its PIN domain. Proc Natl Acad Sci USA 106: 14259-14264, 2009.

12. Fatica A, Tollervey D and Dlakic M: PIN domain of Noblp is required for D-site cleavage in $20 \mathrm{~S}$ pre-rRNA. RNA 10 $1698-1701,2004$

13. Fatica A, Oeffinger M, Dlakic $M$ and Tollervey D: Noblp is required for cleavage of the 3' end of $18 \mathrm{~S}$ rRNA. Mol Cell Biol 23: 1798-1807, 2003.

14. Veith T, Martin R, Wurm JP, et al: Structural and functional analysis of the archaeal endonuclease Nob1. Nucleic Acids Res 40: 3259-3274, 2012.

15. Nazar RN: Ribosomal RNA processing and ribosome biogenesis in eukaryotes. IUBMB Life 56: 457-465, 2004.

16. Montanaro L, Trere D and Derenzini M: Changes in ribosome biogenesis may induce cancer by down-regulating the cell tumor suppressor potential. Biochim Biophys Acta 1825 101-110, 2012.

17. Hershko A and Ciechanover A: The ubiquitin system. Annu Rev Biochem 67: 425-479, 1998.

18. Ferrell K, Wilkinson CR, Dubiel W and Gordon C: Regulatory subunit interactions of the $26 \mathrm{~S}$ proteasome, a complex problem. Trends Biochem Sci 25: 83-88, 2000.

19. Micel LN, Tentler JJ, Smith PG and Eckhardt GS: Role of ubiquitin ligases and the proteasome in oncogenesis: novel targets for anticancer therapies. J Clin Oncol 31: 1231-1238, 2013.

20. Mani A and Gelmann EP: The ubiquitin-proteasome pathway and its role in cancer. J Clin Oncol 23: 4776-4789, 2005.

21. Lin Y, Peng S, Yu H, Teng H and Cui M: RNAi-mediated downregulation of NOB1 suppresses the growth and colony-formation ability of human ovarian cancer cells. Med Oncol 29: 311-317, 2012.

22. Che JP, Li W, Yan Y, et al: Expression and clinical significance of the nin one binding protein and p38 MAPK in prostate carcinoma. Int J Clin Exp Pathol 6: 2300-2311, 2013.

23. Li XY, Luo QF, Li J, et al: Clinical significance of NOB1 expression in breast infiltrating ductal carcinoma. Int J Clin Exp Pathol 6: 2137-2144, 2013.
24. Li Y, Ma C, Qian M, Wen Z, Jing H and Qian D: Downregulation of NOB1 suppresses the proliferation and tumor growth of non-small cell lung cancer in vitro and in vivo. Oncol Rep 31: 1271-1276, 2014

25. Lin S, Meng W, Zhang W, et al: Expression of the NOB1 gene and its clinical significance in papillary thyroid carcinoma. J Int Med Res 41: 568-572, 2013.

26. Huang WY, Chen DH and Ning L: siRNA mediated silencing of NIN1/RPN12 binding protein 1 homolog inhibits proliferation and growth of breast cancer cells. Asian Pac J Cancer Prev 13: 1823-1827, 2012.

27. Lu Z, Guo Q, Shi A, Xie F and Lu Q: Down regulation of NIN/RPN12 binding protein inhibits the growth of human hepatocellular carcinoma cells. Mol Biol Rep 39: 501-507, 2012.

28. Guoan X, Hanning W, Kaiyun C and Hao L: Adenovirusmediated siRNA targeting Mcl-1 gene increases radiosensitivity of pancreatic carcinoma cells in vitro and in vivo. Surgery 147: 553-561, 2010.

29. Yang J, Sun M, Zhang A, Lv C, De W and Wang Z: Adenovirusmediated siRNA targeting $\mathrm{Bcl}-\mathrm{xL}$ inhibits proliferation, reduces invasion and enhances radiosensitivity of human colorectal cancer cells. World J Surg Oncol 9: 117, 2011.

30. Lin CI, Whang EE, Donner DB, et al: Galectin-3 targeted therapy with a small molecule inhibitor activates apoptosis and enhances both chemosensitivity and radiosensitivity in papillary thyroid cancer. Mol Cancer Res 7: 1655-1662, 2009.

31. Tone Y, Tanahashi N, Tanaka K, Fujimuro M, Yokosawa H and Toh-e A: Noblp, a new essential protein, associates with the 26S proteasome of growing saccharomyces cerevisiae cells. Gene 243: 37-45, 2000.

32. Xu G, Bernaudo S, Fu G, Lee DY, Yang BB and Peng C: Cyclin G2 is degraded through the ubiquitin-proteasome pathway and mediates the antiproliferative effect of activin receptor-like kinase 7. Mol Biol Cell 19: 4968-4979, 2008.

33. Fasanaro P, Capogrossi MC and Martelli F: Regulation of the endothelial cell cycle by the ubiquitin-proteasome system. Cardiovasc Res 85: 272-280, 2010.

34. Wang H, Li P and Zhao B: Knockdown of NOB1 expression by RNAi inhibits cellular proliferation and migration in human gliomas. Gene 528: 146-153, 2013.

35. Dhillon AS, Hagan S, Rath O and Kolch W: MAP kinase signalling pathways in cancer. Oncogene 26: 3279-3290, 2007.

36. Yacoub A, Mitchell C, Lebedeva IV, et al: mda-7 (IL-24) Inhibits growth and enhances radiosensitivity of glioma cells in vitro via JNK signaling. Cancer Biol Ther 2: 347-353, 2003.

37. Chang L and Karin M: Mammalian MAP kinase signalling cascades. Nature 410: 37-40, 2001

38. Sofia Vala I, Martins LR, Imaizumi N, et al: Low doses of ionizing radiation promote tumor growth and metastasis by enhancing angiogenesis. PLoS One 5: e11222, 2010.

39. Zhou J, Xu T, Yan Y, et al: MicroRNA-326 functions as a tumor suppressor in glioma by targeting the Nin one binding protein (NOB1). PLoS One 8: e68469, 2013.

40. Wagner EF and Nebreda AR: Signal integration by JNK and p38 MAPK pathways in cancer development. Nat Rev Cancer 9: 537-549, 2009. 